\title{
FATORES ASSOCIADOS AO ESTADO NUTRICIONAL DE IDOSOS
}

Rozana Ferreira Ortiz - Universidade Federal de Ciências da Saúde de Porto Alegre (UFCSPA) - Email: nutri.rozanaortiz@gmail.com

Anny Caroline dos Santos Araujo - Universidade Federal de Ciências da Saúde de Porto Alegre (UFCSPA) Email: annycarolinesaraujo@gmail.com

Me. Bruna Senna Rodrigues - CCG Saúde - Email: brusennar@gmail.com Profa. Dra. Valdeni Terezinha Zani - Universidade Federal de Ciências da Saúde de Porto Alegre (UFCSPA) - Email: valdenizani@ufcspa.edu.br

\section{RESUMO}

Introdução: A população idosa tem sido alvo de diversos estudos científicos, isso ocorre por conta do aumento significativo destes indivíduos que é estimado para as próximas décadas. Faz-se necessário conhecer seu perfil, suas demandas fisiopatológicas, a fim de oferecer um melhor atendimento e cuidado pelos profissionais da saúde. Com isso, o estudo trata-se de uma revisão integrativa com o objetivo de analisar as evidências científicas referentes aos fatores associados ao estado nutricional de idosos no Brasil. Metodologia: A busca de artigos foi realizada nas bases de dados Lilacs e SciELO, onde foram incluídas publicações referentes ao período de 2015 a 2020 , onde os artigos selecionados foram avaliados conforme Classificação Hierárquica de Evidências. Resultados: Foram selecionados 14 artigos, onde os resultados demonstraram que idosos com excesso de peso possuiam relação positiva com doenças crônicas não-transmissíveis como hipetensão arterial e dislipidemia, além da renda per capta e escolaridade mínima de 8 anos. A maioria eram mulheres e residiam nas regiões Sul e Sudeste do Brasil, além da relação com estado civil, como idosos viúvos, além de possuir saneamento básico. Os idosos residentes de estrato rural, que possuiam depressão e idade mais avançada tiveram maiores chances de risco nutricional ou baixo peso. Conclusão: Conclui-se que, os fatores que mais influenciaram o estado nutricional dos idosos foram doenças cronicas não-transmissíveis, renda e idade. Além disso, idosos que eram menos vulneráveis e residentes de instituições possuiam um estado nutricional adequado e menoes chances de risco nutricional, devido ao acesso à saude e cuidados proffisionais.

Palavras-chave: Estado nutricional (Nutritional status); Idosos (Elderly). 